\title{
Transport Coefficients of Poly(diisopropyl fumarate) in Dilute Solution
}

\author{
By Masayuki NAKATSUJI, Kouta SOUTOKU, Masashi OSA, and Takenao YOSHIZAKI*
}

The intrinsic viscosity $[\eta]$ was determined for 13 samples of poly(diisopropyl fumarate), each with the fraction of racemo diads $f_{\mathrm{r}}=0.22$, in the range of weight-average molecular weight $M_{\mathrm{w}}$ from $4.02 \times 10^{4}$ to $8.59 \times 10^{5}$ in tetrahydrofuran at $30.0^{\circ} \mathrm{C}$. The translational diffusion coefficient $D$ was also determined from dynamic light scattering measurements for 12 of them in the range of $M_{\mathrm{w}}$ from $4.95 \times 10^{4}$ to $8.59 \times 10^{5}$ under the same solvent condition. The data so obtained were analyzed on the basis of the corresponding transport theories for the unperturbed Kratky-Porod (KP) wormlike cylinder model combined with the quasi-two-parameter theory for the intramolecular excluded-volume effect. It is shown that the experimental values are in quantitative agreement with the perturbed KP theory ones by the use of the model parameter values consistent with those determined previously from analyses of the mean-square radius of gyration $\left\langle S^{2}\right\rangle$ and second virial coefficient $A_{2}$. An examination was also made of behavior of the Flory-Fox factor $\Phi$ and reduced hydrodynamic radius $\rho^{-1}$ as functions of $M_{\mathrm{w}}$.

KEY WORDS: Poly(diisopropyl fumarate) / Intrinsic Viscosity / Translational Diffusion Coefficient / Kratky-Porod Wormlike Chain Model / Excluded-Volume Effect / Quasi-Two-Parameter Theory /

In a previous paper, ${ }^{1}$ we have reported experimental results for the mean-square radius of gyration $\left\langle S^{2}\right\rangle$ and second virial coefficient $A_{2}$ of poly(diisopropyl fumarate) (PDiPF) with the fraction of racemo diads $f_{\mathrm{r}}=0.22$ in tetrahydrofuran (THF) at $30.0^{\circ} \mathrm{C}$ (good solvent). From analyses of the results on the basis of the Kratky-Porod (KP) wormlike chain model ${ }^{2,3}$ with the intra- and intermolecular excluded-volume effects, ${ }^{3}$ it has then been shown that the unperturbed KP theory ${ }^{4}$ of $\left\langle S^{2}\right\rangle$ combined with the quasi-two-parameter (QTP) theory ${ }^{3,5-7}$ and the Yamakawa theory ${ }^{8}$ of $A_{2}$ may quantitatively reproduce those results if the parameter values $113 \AA, 89 \AA^{-1}$, and 0.097 are used for the stiffness parameter $\lambda^{-1}$, the shift factor $M_{\mathrm{L}}$ as defined as the molecular weight per unit contour length of the $\mathrm{KP}$ chain, and the reduced excluded-volume strength $\lambda B$, respectively. We note that the $M_{\mathrm{L}}$ value $89 \AA^{-1}$ so determined is consistent with the value $80.3-84.7 \AA^{-1}$ estimated on the basis of reasonable local chain conformations of PDiPF. ${ }^{1}$

Because of steric hindrance to internal rotations caused by bulky substituents, i.e., ester isopropyl groups, on every main-chain carbon atom, the PDiPF chain was expected to be stiff. Its $\lambda^{-1}$ is, however, not so large as that for a typical semiflexible polymer such as $\operatorname{poly}(n$-hexyl isocyanate) (PHIC) $\left(\lambda^{-1}=840 \AA\right),{ }^{9}$ although it is, of course, remarkably larger than that for a typical flexible polymer such as atactic polystyrene (a-PS) $\left(\lambda^{-1}=20.6 \AA\right){ }^{3,10}$ The main assertion made in the previous paper $^{1}$ is that the excluded-volume effects must be taken into account in analyses of dilute solution properties of PDiPF (having moderate chain stiffness) in THF at $30.0^{\circ} \mathrm{C}$. As a continuation of the previous paper, ${ }^{1}$ we here determine the intrinsic viscosity $[\eta]$ and translational diffusion coefficient $D$ (or the hydrodynamic radius $R_{\mathrm{H}}$ defined from $D$ ) from viscosity and dynamic light scattering (DLS) measurements, respectively, for PDiPF in the same solvent condition as that in the previous paper, ${ }^{1}$ and confirm the assertion by analyzing the results so obtained on the basis of corresponding theories for the unperturbed KP wormlike cylinder combined with the QTP theory. ${ }^{3}$

In addition to the above-mentioned main purpose of the present paper, we also examine behavior of the Flory-Fox factor $\Phi$ and also of the reduced hydrodynamic radius $\rho^{-1}$, which may be calculated from $[\eta]$ and $\left\langle S^{2}\right\rangle$ and from $R_{\mathrm{H}}$ and $\left\langle S^{2}\right\rangle$, respectively. It is well known that both $\Phi$ and $\rho^{-1}$ are sensitive to chain stiffness, ${ }^{3}$ so that the examination may be a fair judge of whether the previous estimation of $\lambda^{-1}$ is reasonable or not.

\section{EXPERIMENTAL}

\section{Materials}

All the PDiPF samples used in this study are the same as those used in the previous study ${ }^{1}$ of $\left\langle S^{2}\right\rangle$ and $A_{2}$, i.e., fractions separated by fractional precipitation from the original samples prepared by radical polymerization following the procedure of Otsu et al. ${ }^{11,12}$ and Matsumoto and Nakagawa. ${ }^{13}$ The values of the weight-average molecular weight $M_{\mathrm{w}}$, the ratio $M_{\mathrm{w}} / M_{\mathrm{n}}$ of $M_{\mathrm{w}}$ to the number-average molecular weight $M_{\mathrm{n}}$, and $f_{\mathrm{r}}$ are given in Table I, which have been reproduced from Table I of ref 1. The samples were freeze-dried from their benzene solutions after filtration through a Teflon membrane of pore size $1.0 \mu \mathrm{m}$ prior to use.

The solvent THF used for viscosity and DLS measurements was purified by distillation after refluxing over sodium. 
Table I. Values of $M_{\mathrm{w}}, M_{\mathrm{w}} / M_{\mathrm{n}}$, and $f_{\mathrm{r}}$ for Poly(diisopropyl fumarate)

\begin{tabular}{lccc}
\hline sample & $M_{\mathrm{w}}$ & $M_{\mathrm{w}} / M_{\mathrm{n}}$ & $f_{\mathrm{r}}$ \\
\hline PDiPF4 $^{\mathrm{a}}$ & $4.02 \times 10^{4}$ & & \\
PDiPF5 & $4.95 \times 10^{4}$ & 1.10 & 0.22 \\
PDiPF6 & $6.31 \times 10^{4}$ & 1.05 & \\
PDiPF8 & $8.00 \times 10^{4}$ & 1.10 & \\
PDiPF9 & $9.17 \times 10^{4}$ & 1.07 & \\
PDiPF15 & $1.53 \times 10^{5}$ & 1.08 & \\
PDiPF18 & $1.83 \times 10^{5}$ & 1.08 & \\
PDiPF22 & $2.18 \times 10^{5}$ & 1.09 & 0.22 \\
PDiPF34 & $3.37 \times 10^{5}$ & 1.07 & \\
PDiPF44 & $4.37 \times 10^{5}$ & 1.07 & \\
PDiPF50 & $5.00 \times 10^{5}$ & 1.05 & \\
PDiPF62 & $6.20 \times 10^{5}$ & 1.05 & \\
PDiPF86 & $8.59 \times 10^{5}$ & & \\
\hline
\end{tabular}

${ }^{\text {a All values of }} M_{\mathrm{w}}$ had been determined from light scattering in THF at $30.0^{\circ} \mathrm{C}$, all values of $M_{\mathrm{w}} / M_{\mathrm{n}}$ from analytical gel permeation chromatography, and all values of $f_{\mathrm{r}}$ from ${ }^{13} \mathrm{C}$ NMR. ${ }^{1}$

\section{Viscosity}

Viscosity measurements were carried out for all the samples listed in Table I in THF at $30.0^{\circ} \mathrm{C}$. For the measurements, we used conventional capillary and four-bulb spiral capillary viscometers of the Ubbelohde type. The flow time was measured to a precision of $0.1 \mathrm{~s}$, keeping the difference between those of the solvent and solution larger than $20 \mathrm{~s}$. The test solutions were maintained at $30.0^{\circ} \mathrm{C}$ within $\pm 0.005^{\circ} \mathrm{C}$ during the measurements.

The test solutions were prepared gravimetrically and made homogeneous by continuous stirring in the dark at room temperature for $2 \mathrm{~d}$. The polymer mass concentrations $c$ were calculated from the weight fractions with the densities of the solutions. The density of each solution was calculated with the partial specific volume $v_{2}$ of the polymer and the density $\rho_{0}$ of the solvent. The quantities $v_{2}$ and $\rho_{0}$ were measured with a pycnometer of the Lipkin-Davison type having a volume of $3 \mathrm{~cm}^{3}$. The values of $v_{2}$ so determined in THF at $30.0^{\circ} \mathrm{C}$ are $0.873 \mathrm{~cm}^{3} / \mathrm{g}$ for all the samples independently of $M_{\mathrm{w}}$. The value of $\rho_{0}$ of $\mathrm{THF}$ at $30.0^{\circ} \mathrm{C}$ is $0.8751 \mathrm{~g} / \mathrm{cm}^{3}$. Density corrections were also made in the calculations of the relative viscosity $\eta_{\mathrm{r}}$ from the flow times of the solution and solvent. The data obtained for the specific viscosity $\eta_{\mathrm{sp}}$ and $\eta_{\mathrm{r}}$ were treated as usual by the Huggins $\left(\eta_{\mathrm{sp}} / c\right.$ vs. $\left.c\right)$ and Fuoss-Mead $\left(\ln \eta_{\mathrm{r}} / c\right.$ vs. $c$ ) plots, respectively, to determine $[\eta]$ and the Huggins coefficient $k^{\prime}$.

\section{Dynamic Light Scattering}

DLS measurements were carried out to determine $D$ for all the samples except PDiPF4 in THF at $30.0^{\circ} \mathrm{C}$ by the use of a Brookhaven Instruments model BI-200SM light scattering goniometer with vertically polarized incident light of wavelength $\lambda_{0}=532 \mathrm{~nm}$ (in a vacuum) from a Spectra-Physics model Millenia Pro 2s Nd: $\mathrm{VVO}_{4}$ laser. The photomultiplier tube used was EMI 9893B/350, the output from which was processed by a Brookhaven Instruments model BI-9000AT Digital Correlator. (An electric shutter was attached to the original detector alignment in order to monitor the dark count automatically.) The normalized autocorrelation function $g^{(2)}(t)$ of scattered light intensity $I(t)$ at time $t$ was measured at 3-5 concentrations and at scattering angles $\theta$ ranging from 22 to $46^{\circ}$. The most concentrated solution of each sample was prepared in the same manner as in the case of the viscosity measurements. It was optically purified by filtration through a Teflon membrane of pore size 0.45 or $0.10 \mu \mathrm{m}$. The solutions of lower concentrations were obtained by successive dilution. The polymer mass concentrations $c$ were calculated from the weight fractions with the densities of the solutions.

From the data for $g^{(2)}(t)$ so determined at finite concentrations $c$, we determined $D$ at an infinitely long time at infinite dilution in the same manner as that used in the previous studies. ${ }^{14,15}$ At small $c$, the plot of $(1 / 2) \ln \left[g^{(2)}(t)-1\right]$ against $t$ in general follows a straight line represented by

$$
(1 / 2) \ln \left[g^{(2)}(t)-1\right]=\text { const }-A t
$$

with $A$ the slope for such large $t$ that all the internal motions of solute polymer chains have relaxed away.

With the slope $A$ evaluated from the plot, we may determine the apparent diffusion coefficient $D^{(\mathrm{LS})}(c)$ at finite $c$ from

$$
D^{(\mathrm{LS})}(c)=\lim _{k \rightarrow 0} A / k^{2}
$$

where $k$ is the scattering vector and is given by

$$
k=\left(4 \pi n_{0} / \lambda_{0}\right) \sin (\theta / 2)
$$

with $n_{0}$ the refractive index of the solvent. At very small $c$, $D^{(\mathrm{LS})}(c)$ may be expanded as

$$
D^{(\mathrm{LS})}(c)=D^{(\mathrm{LS})}(0)\left(1+k_{\mathrm{D}}^{(\mathrm{LS})} c+\cdots\right)
$$

so that the desired $D=D(\infty)$ (at an infinitely long time) may be determined by an extrapolation of $D^{(\mathrm{LS})}(c)$ to $c=0$ as

$$
D=D^{(\mathrm{LS})}(0)
$$

The values of $n_{0}$ at $\lambda_{0}=532 \mathrm{~nm}$ and of the viscosity coefficient $\eta_{0}$ for THF at $30.0^{\circ} \mathrm{C}$ are $1.404_{5}$ and $0.439_{7} \mathrm{cP}$, respectively. The former value was estimated by a linear interpolation of the plot of $n_{0}$ against $\lambda_{0}^{-2}$ with the values $1.410_{8}$ and $1.403_{8}$ of $n_{0}$ at $\lambda_{0}=436$ and $546 \mathrm{~nm}$, respectively, measured by the use of an Abbe refractometer (ERMA OPTICAL WORKS).

\section{RESULTS}

\section{Intrinsic Viscosity $[\eta]$}

Intrinsic viscosity data for all the PDiPF samples in THF at $30.0^{\circ} \mathrm{C}$ are summarized in Table II along with the values of the Huggins coefficient $k^{\prime}$. It is seen that $k^{\prime}$ is almost independent of $M_{\mathrm{w}}$ and its value $c a$. 0.4 is somewhat larger than that for typical flexible chains having large $M_{\mathrm{W}}$ in good solvents. ${ }^{16-20}$

Figure 1 shows double-logarithmic plots of $[\eta]$ (in $\mathrm{dL} / \mathrm{g}$ ) against $M_{\mathrm{w}}$ for PDiPF in THF at $30.0^{\circ} \mathrm{C}$. The unfilled circles represent the present experimental values. The present data points are seen to form a curve slightly convex upward, its slope being $c a$. 0.86 for $M_{\mathrm{w}} \lesssim 2 \times 10^{5}$ and $c a$. 0.73 for $M_{\mathrm{w}} \gtrsim 3 \times 10^{5}$. The rather steep slope in the range of $M_{\mathrm{w}} \lesssim$ $2 \times 10^{5}$ may be due to effects of chain stiffness, as in the case 
Table II. Results of Viscosity Measurements for Poly(diisopropyl fumarate) in THF at $30.0^{\circ} \mathrm{C}$

\begin{tabular}{lll}
\hline sample & $\begin{array}{c}{[\eta]} \\
(\mathrm{dL} / \mathrm{g})\end{array}$ & $k^{\prime}$ \\
\hline PDiPF4 & 0.308 & 0.41 \\
PDiPF5 & 0.349 & 0.44 \\
PDiPF6 & 0.455 & 0.42 \\
PDiPF8 & 0.541 & 0.44 \\
PDiPF9 & 0.589 & 0.42 \\
PDiPF15 & 0.874 & 0.43 \\
PDiPF18 & 1.01 & 0.42 \\
PDiPF22 & 1.13 & 0.42 \\
PDiPF34 & 1.65 & 0.42 \\
PDiPF44 & 1.98 & 0.36 \\
PDiPF50 & 2.38 & 0.42 \\
PDiPF62 & 2.62 & 0.40 \\
PDiPF86 & 3.27 & 0.42 \\
\hline
\end{tabular}

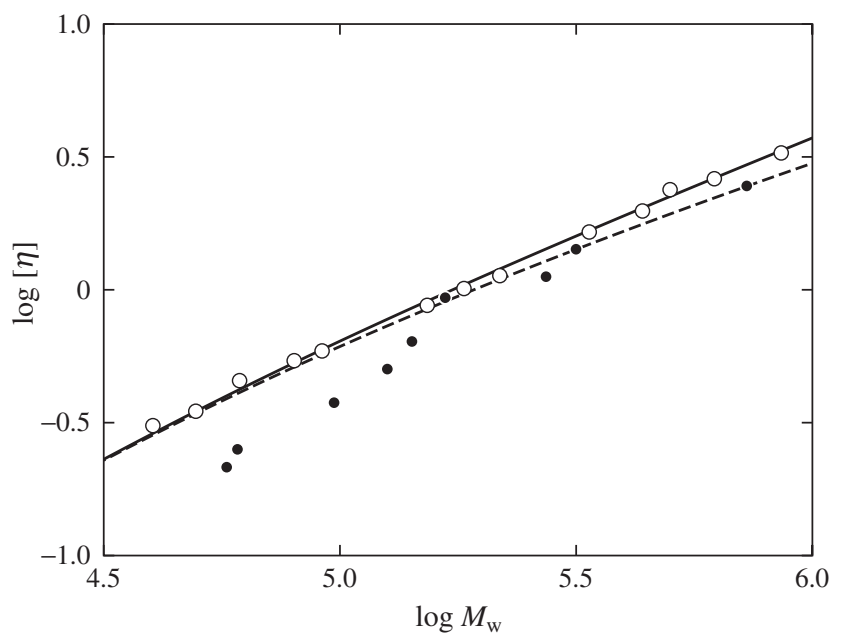

Figure 1. Double-logarithmic plots of $[\eta]$ (in $\mathrm{dL} / \mathrm{g}$ ) against $M_{\mathrm{w}}$ for PDiPF in THF at $30.0^{\circ} \mathrm{C}$ : (O) present data; $(\bigcirc)$ data by Matsumoto and Nakagawa. ${ }^{13}$ The solid and dashed curves represent the best-fit $\mathrm{KP}$ theory values with and without excluded volume, respectively (see the text).

of $\left\langle S^{2}\right\rangle .{ }^{1}$ In the figure, the solid and dashed curves represent the KP theory values with and without excluded volume, respectively, which are discussed in the next (DISCUSSION) section.

The figure also includes literature data for the same polymer-solvent system reported by Matsumoto and Nakagawa $^{13}$ (filled circles). The literature and present values are rather in good agreement in the range of $M_{\mathrm{w}} \gtrsim 2 \times 10^{5}$, while the former deviate downward from the latter for $M_{\mathrm{w}} \lesssim 2 \times 10^{5}$. The reason for this deviation is not clear.

\section{Translational Diffusion Coefficient $D$ and Hydrodynamic Radius $\boldsymbol{R}_{\mathbf{H}}$}

The data points $(1 / 2) \ln \left[g^{(2)}(t)-1\right]$ as a function of $t$ directly obtained from the DLS measurements for each test solution at each scattering angle $\theta$ were found to follow a straight line, so that the slope $A$ on the right-hand side in eq 1 could be unambiguously determined. Further, in the range of $k$ ( or $\theta$ ) where the DLS measurements were carried out, the ratio

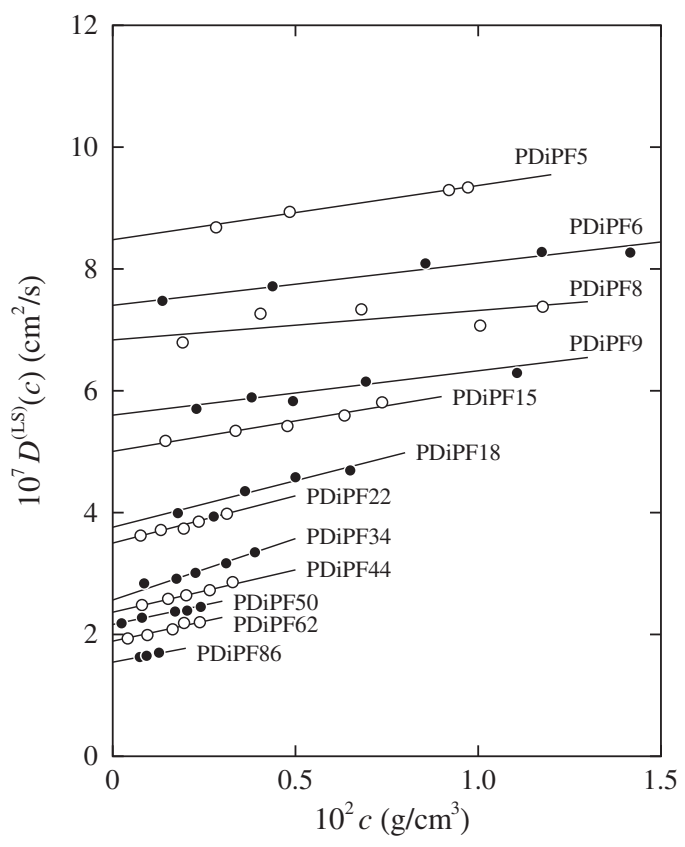

Figure 2. Plots of $D^{(\mathrm{LS})}(c)$ against $c$ for the indicated PDiPF samples in THF at $30.0^{\circ} \mathrm{C}$.

Table III. Results of DLS Measurements for Poly(diisopropyl fumarate) in $\mathrm{THF}$ at $30.0^{\circ} \mathrm{C}$

\begin{tabular}{lccc}
\hline sample & $\begin{array}{c}10^{7} D \\
\left(\mathrm{~cm}^{2} / \mathrm{s}\right)\end{array}$ & $\begin{array}{c}k_{\mathrm{D}}^{(\mathrm{LS})} \\
\left(\mathrm{cm}^{3} / \mathrm{g}\right)\end{array}$ & $\begin{array}{c}R_{\mathrm{H}} \\
(\AA)\end{array}$ \\
\hline PDiPF5 & 8.48 & 11 & 59.6 \\
PDiPF6 & 7.40 & 9.4 & 68.2 \\
PDiPF8 & 6.84 & 7.1 & 73.8 \\
PDiPF9 & 5.63 & 13 & 89.7 \\
PDiPF15 & 5.01 & 20 & 101 \\
PDiPF18 & 3.76 & 41 & 134 \\
PDiPF22 & 3.50 & 45 & 144 \\
PDiPF34 & 2.57 & 78 & 196 \\
PDiPF44 & 2.37 & 60 & 213 \\
PDiPF50 & 2.16 & 59 & 233 \\
PDiPF62 & 1.89 & 69 & 267 \\
PDiPF86 & 1.55 & 74 & 327
\end{tabular}

$A / k^{2}$ was independent of $k$ within experimental error for all the test solutions, although we do not show the results here. Then $D^{(\mathrm{LS})}(c)$ for each solution was determined as a mean of the observed values of $A / k^{2}$ over $k$.

Figure 2 shows plots of $D^{(\mathrm{LS})}(c)$ so obtained against $c$ for the indicated PDiPF samples in $\mathrm{THF}$ at $30.0^{\circ} \mathrm{C}$. The data points for each sample are seen to follow a straight line, and therefore $D^{(\mathrm{LS})}(0)(=D)$ and $k_{\mathrm{D}}^{(\mathrm{LS})}$ on the right-hand side in eq 4 may be accurately determined from its ordinate intercept and slope, respectively. The values of $D$ and $k_{\mathrm{D}}^{(\mathrm{LS})}$ so obtained for PDiPF in $\mathrm{THF}$ at $30.0^{\circ} \mathrm{C}$ are given in Table III. The estimated error in $D$ is $\pm 3 \%$ at most. In the last column of the Table are also given the values of the hydrodynamic radius $R_{\mathrm{H}}$ calculated from the defining equation

$$
R_{\mathrm{H}}=k_{\mathrm{B}} T / 6 \pi \eta_{0} D
$$




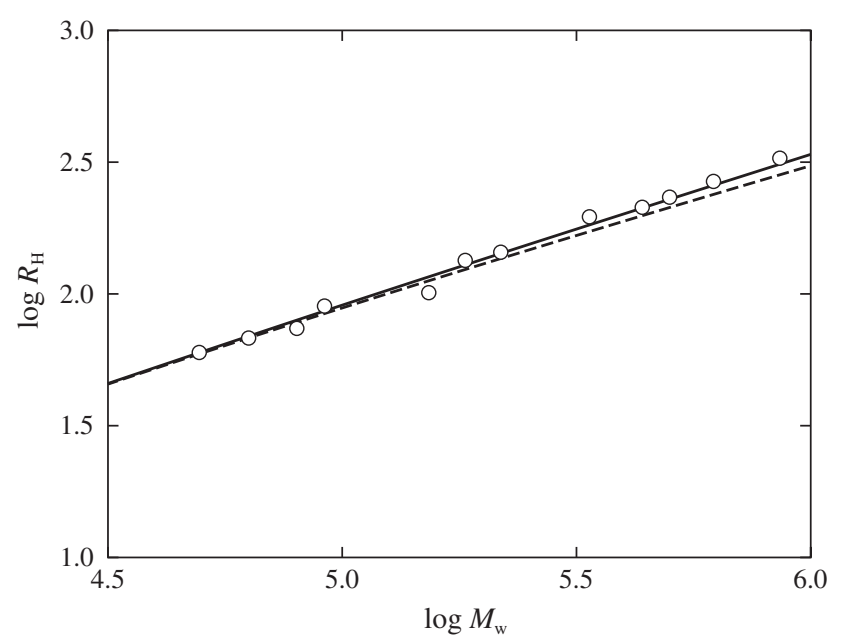

Figure 3. Double-logarithmic plots of $R_{\mathrm{H}}$ (in $\AA$ ) against $M_{\mathrm{W}}$ for PDiPF in THF at $30.0^{\circ} \mathrm{C}$. The solid and dashed curves represent the best-fit KP theory values with and without excluded volume, respectively (see the text).

where $k_{\mathrm{B}}$ is the Boltzmann constant, $T$ is the absolute temperature, and $\eta_{0}$ is the viscosity coefficient of the solvent. The quantity $k_{\mathrm{D}}^{(\mathrm{LS})}$ is positive for all the samples examined and increases with increasing $M_{\mathrm{w}}$, as in the cases of typical flexible polymers having large $M_{\mathrm{W}}$ in good solvents. ${ }^{19-23}$

In Figure $3, R_{\mathrm{H}}$ (in $\AA$ ) calculated from eq 6 with the present experimental values of $D$ given in the second column of Table III are double-logarithmically plotted against $M_{\mathrm{w}}$. The data points $(\bigcirc)$ are seen to form a curve slightly convex upward, its slope being $c a$. 0.62 for $M_{\mathrm{w}} \lesssim 2 \times 10^{5}$ and $c a .0 .58$ for $M_{\mathrm{w}} \gtrsim 3 \times 10^{5}$. We note that the error bounds to each value of $R_{\mathrm{H}}$ do not exceed the radius of the symbols in the figure. The rather steep slope in the range of $M_{\mathrm{w}} \lesssim 2 \times 10^{5}$ may be due to the effect of chain stiffness of PDiPF, as in the cases of $[\eta]$ shown in Figure 1 and also of $\left\langle S^{2}\right\rangle .{ }^{1}$ In the figure, the solid and dashed curves represent the KP theory values with and without excluded volume, which are discussed in the next (DISCUSSION) section.

\section{DISCUSSION}

\section{Analysis of $[\eta]$ on the Basis of the KP Model}

We first analyze the data for $[\eta]$ on the basis of the unperturbed KP cylinder model ${ }^{3,24,25}$ combined with the QTP theory. ${ }^{3,5-7}$ We note that the KP chain model having only a bending energy is a special case of the helical wormlike (HW) chain model having both bending and torsional energies ${ }^{3}$ and that the QTP theory has been constructed on the basis of the latter model.

For the unperturbed KP cylinder model of total contour length $L$ and diameter $d$, $[\eta]_{0}$ may be written in the form,

$$
[\eta]_{0}=\frac{1}{\lambda^{2} M_{\mathrm{L}}} f_{\eta, \mathrm{KP}}(\lambda L ; \lambda d)
$$

where the subscript 0 on $[\eta]$ denotes the unperturbed value and $f_{\eta, \mathrm{KP}}$ as a function of the reduced contour length $\lambda L$ and reduced diameter $\lambda d$ is defined by

$$
f_{\eta, \mathrm{KP}}(\lambda L ; \lambda d)=\lambda^{-1} M_{\mathrm{L}}[\eta]_{\mathrm{KP}}
$$

with $[\eta]_{\mathrm{KP}}$ being given by eq 6.89 with eq 6.90 of ref 3 (or eq 23 with eq 24 of ref 25 ) for $\lambda L \geq 2.278$ and by eq 6.91 with eqs 6.92 and 6.94 of ref 3 (or eq 25 with eqs 26 and 32 of ref 25$)$ for $\lambda L<2.278$. We note that $[\eta]_{\mathrm{KP}}$ in refs 3 and 25 is written in units of $\left(\lambda^{-1}\right)^{3}$ and the quantities $L$ and $d$ in those references mean the reduced quantities $\lambda L$ and $\lambda d$, respectively. In the limit of $\lambda L \rightarrow \infty, f_{\eta, \mathrm{KP}}$ satisfies the following asymptotic relation,

$$
\lim _{\lambda L \rightarrow \infty} \frac{f_{\eta, \mathrm{KP}}(\lambda L ; \lambda d)}{(\lambda L)^{1 / 2}}=\Phi_{\infty}
$$

where $\Phi_{\infty}$ denotes the (theoretical) coil-limiting value $2.87 \times$ $10^{23} \mathrm{~mol}^{-1}$ of the Flory-Fox factor $\Phi=M[\eta] /\left(6\left\langle S^{2}\right\rangle\right)^{3 / 2}$ with $M$ the molecular weight (for the unperturbed chain in the limit of $\lambda L \rightarrow \infty)$. We note that $\Phi$ is the reduced hydrodynamic volume $^{3}$ defined as the ratio of the hydrodynamic (molar) volume $V_{\mathrm{H}}$ to $\left\langle S^{2}\right\rangle^{3 / 2}$,

$$
\Phi=V_{\mathrm{H}} /\left\langle S^{2}\right\rangle^{3 / 2}
$$

with $V_{\mathrm{H}}$ defined from $[\eta]$ as

$$
V_{\mathrm{H}}=6^{-3 / 2} M[\eta]
$$

In a comparison of theory with experiment, $L$ may be related to $M$ by

$$
L=M / M_{\mathrm{L}}
$$

For the perturbed KP cylinder model, $[\eta]$ may conventionally be expressed as a product of $[\eta]_{0}$ given by eq 7 and the cube of the viscosity-radius expansion factor $\alpha_{\eta}$,

$$
[\eta]=[\eta]_{0} \alpha_{\eta}^{3}
$$

In the framework of the QTP scheme or the YamakawaStockmayer-Shimada theory, ${ }^{3,5-7}$ all the expansion factors, including $\alpha_{\eta}$, are functions only of the scaled intramolecular excluded-volume parameter $\tilde{z}$ defined by

$$
\tilde{z}=\frac{3}{4} K(\lambda L) z
$$

where $z$ is the (conventional) excluded-volume parameter $z$ appearing in the two-parameter (TP) theory ${ }^{26}$ and is defined by

$$
z=(3 / 2 \pi)^{3 / 2}(\lambda B)(\lambda L)^{1 / 2}
$$

with $B$ the excluded-volume strength. We note that in the case of the KP chain, $B$ may be written in terms of the binary-cluster integral $\beta$ between beads (or statistical segments) and their spacing $a$. We omit here the explicit expression for $B$, since it is unnecessary for the following discussion. In eq 14 , the coefficient $K(L)$ as a function of reduced $L$ is given by

$$
\begin{array}{rr}
K(L)=\frac{4}{3}-2.711 L^{-1 / 2}+\frac{7}{6} L^{-1} & \text { for } L>6 \\
=L^{-1 / 2} \exp \left(-6.611 L^{-1}+0.9198+\right. & 0.03516 L) \\
& \text { for } L \leq 6
\end{array}
$$


It is seen from eq 14 with eq 16 that $\tilde{z}=z$ in the limit of $\lambda L \rightarrow \infty$ (random-coil limit), so that the QTP theory becomes identical with the TP theory in this limit. We note that the function $K(L)$ represents the effects of chain stiffness on the intramolecular excluded-volume effect. As usual, ${ }^{3}$ we adopt the Barrett equation $^{27}$ for $\alpha_{\eta}$,

$$
\alpha_{\eta}=\left(1+3.8 \tilde{z}+1.9 \tilde{z}^{2}\right)^{0.1}
$$

From eq 13 with eqs 7, 12, and 14-17, it is seen that the perturbed KP theory value of $[\eta]$ may be calculated as a function of $M$ for a given set of values of the four parameters $\lambda^{-1}, M_{\mathrm{L}}$, $\lambda B$, and $d$. In other words, we can in principle determine the four parameters for PDiPF from a comparison of theory with experiment. Unfortunately, however, the plot of $[\eta]$ against $M_{\mathrm{w}}$ for PDiPF shown in Figure 1 has no salient feature such as a $\mathrm{S}$ shaped curve observed for PHIC, ${ }^{9}$ so that it is difficult to determine all of them from the comparison. We therefore assume here the values $113 \AA$ and 0.097 of $\lambda^{-1}$ and $\lambda B$, respectively, determined previously ${ }^{1}$ from the analyses of $\left\langle S^{2}\right\rangle$ and $A_{2}$ and then determine the remaining two parameters $M_{\mathrm{L}}$ and $d$.

In Figure 1, the solid curve associated with the present data points $(\bigcirc)$ represents the best-fit perturbed KP theory values calculated from eq 13 with eqs 7, 12, and 14-17 with the values $92 \AA^{-1}$ and $15 \AA$ of $M_{\mathrm{L}}$ and $d$, respectively. Although the $M_{\mathrm{L}}$ value $92 \AA^{-1}$ so determined is slightly (ca. 3\%) larger than $89 \AA^{-1}$ determined previously ${ }^{1}$ from $\left\langle S^{2}\right\rangle$ and $A_{2}$, the quantitative agreement between theory and experiment confirms the previous assertion. ${ }^{1}$ The $d$ value $15 \AA$ is consistent with $c a$. $13 \AA$ estimated from reasonable local chain conformations of PDiPF (see Figure 3 in ref 1).

In Figure 1, the dashed curve represents the unperturbed KP theory values calculated from eq 7 with eq 12 with the abovementioned values of $\lambda^{-1}, M_{\mathrm{L}}$, and $d$. It is seen that the experimental value $(\bigcirc)$ and also the perturbed KP theory one (solid curve) agree with the unperturbed KP theory value for $M_{\mathrm{w}} \lesssim 10^{5}$ but deviate upward progressively from the latter with increasing $M_{\mathrm{w}}$ for $M_{\mathrm{w}} \gtrsim 10^{5}$ due to the intramolecular excluded-volume effect, as in the case of $\left\langle S^{2}\right\rangle{ }^{1}$

\section{Analysis of $D$ on the Basis of the KP Model}

For the unperturbed KP cylinder model of total contour length $L$ and diameter $d, D_{0}$ may be written in the form, $3,28,29$

$$
D_{0}=\left(\frac{k_{\mathrm{B}} T}{3 \pi \eta_{0} L}\right) f_{\mathrm{D}, \mathrm{KP}}(\lambda L ; \lambda d)
$$

where the subscript 0 on $D$ denotes the unperturbed value and $f_{\mathrm{D}, \mathrm{KP}}$ as a function of $\lambda L$ and $\lambda d$ is given by eqs $6.41-6.43$ with eqs 6.22 and 6.23 of ref 3 . We note that the quantity $3 \pi \eta_{0} L / \Xi$ given by eqs 49 and 51 in ref 28 or by eq 14 in ref 29 with $c_{\infty}=1$ corresponds to $f_{\mathrm{D}, \mathrm{KP}}$ and that in refs 3,28 and 29 , the quantities $L$ and $d$ mean the reduced quantities $\lambda L$ and $\lambda d$, respectively. Then the hydrodynamic radius $R_{\mathrm{H}, 0}$ of the unperturbed KP chain may be written in terms of $f_{\mathrm{D}, \mathrm{KP}}$ as

$$
R_{\mathrm{H}, 0}=\frac{L}{2 f_{\mathrm{D}, \mathrm{KP}}(\lambda L ; \lambda d)}
$$

In the limit of $\lambda L \rightarrow \infty, f_{\mathrm{D}, \mathrm{KP}}$ satisfies the following asymptotic relation,

$$
\lim _{\lambda L \rightarrow \infty} \frac{f_{\mathrm{D}, \mathrm{KP}}(\lambda L ; \lambda d)}{(\lambda L)^{1 / 2}}=\frac{\sqrt{6}}{2} \rho_{\infty}
$$

where $\rho_{\infty}$ denotes the (Kirkwood theoretical) coil-limiting value 1.505 of the ratio $\rho$ of $\left\langle S^{2}\right\rangle^{1 / 2}$ to $R_{\mathrm{H}}$ (for the unperturbed chain in the limit of $\lambda L \rightarrow \infty$ ). We note that the inverse $\rho^{-1}$ of $\rho$ is called the reduced hydrodynamic radius ${ }^{3}$ defined as the ratio of $R_{\mathrm{H}}$ to $\left\langle S^{2}\right\rangle^{1 / 2}$,

$$
\rho^{-1}=R_{\mathrm{H}} /\left\langle S^{2}\right\rangle^{1 / 2}
$$

For the perturbed KP cylinder model, $R_{\mathrm{H}}$ may be expressed as a product of $R_{\mathrm{H}, 0}$ and the hydrodynamic-radius expansion factor $\alpha_{\mathrm{H}}$,

$$
R_{\mathrm{H}}=R_{\mathrm{H}, 0} \alpha_{\mathrm{H}}
$$

In the framework of the QTP scheme, ${ }^{3,5-7} \alpha_{\mathrm{H}}$ is also a function only of $\tilde{z}$ and may be written in the form, 3,30

$$
\alpha_{\mathrm{H}}=\alpha_{\mathrm{H}}^{(0)} h_{\mathrm{H}}
$$

where we use the Barrett equation ${ }^{31}$ for $\alpha_{\mathrm{H}}^{(0)}$,

$$
\alpha_{\mathrm{H}}^{(0)}=\left(1+5.93 \tilde{z}+3.59 \tilde{z}^{2}\right)^{0.1}
$$

and $h_{\mathrm{H}}$, which represents a possible effect of fluctuating hydrodynamic interaction on $\alpha_{\mathrm{H}},{ }^{30}$ is given by

$$
h_{\mathrm{H}}=\frac{0.88}{1-0.12 \alpha_{S}^{-0.43}}
$$

In eq 25 , we use the Domb-Barrett equation ${ }^{32}$ for the gyrationradius expansion factor $\alpha_{S}$,

$$
\begin{aligned}
\alpha_{S}^{2}= & {\left[1+10 \tilde{z}+(70 \pi / 9+10 / 3) \tilde{z}^{2}+8 \pi^{3 / 2} \tilde{z}^{3}\right]^{2 / 15} } \\
& \times\left[0.933+0.067 \exp \left(-0.85 \tilde{z}-1.39 \tilde{z}^{2}\right)\right]
\end{aligned}
$$

We note that in eq 24 the coefficient 5.93 of $\tilde{z}$ has been adopted in place of the coefficient 6.09 originally adopted by Barrett. ${ }^{3}$

From eq 22 with eqs 12,19 , and $23-26$, it is seen that $R_{\mathrm{H}}$ may also be calculated as a function of $M$ for a given set of values of the four parameters $\lambda^{-1}, M_{\mathrm{L}}, \lambda B$, and $d$. As in the case of $[\eta]$, we assume the values $113 \AA$ and 0.097 of $\lambda^{-1}$ and $\lambda B$, respectively, and determine $M_{\mathrm{L}}$ and $d$ from a comparison of theory with experiment. In Figure 3, the solid curve represents the best-fit perturbed KP theory values calculated from eq 22 with eqs 12,19 , and 23-26 with the values $81 \AA^{-1}$ and $16 \AA$ of $M_{\mathrm{L}}$ and $d$, respectively. It is seen from the figure that the theory may quantitatively explain the behavior of the experimental results. The $M_{\mathrm{L}}$ value $81 \AA^{-1}$ so determined from $D$ is somewhat smaller than $92 \AA^{-1}$ determined above from $[\eta]$ and $89 \AA^{-1}$ determined previously ${ }^{1}$ from $\left\langle S^{2}\right\rangle$ and $A_{2}$. This tendency is the same as that in the cases of flexible polymers in the $\Theta$ solvents previously ${ }^{3}$ studied (see Tables 5.1 and 6.3 of ref 3). As often mentioned, ${ }^{3,14}$ it may be regarded as arising from the disagreement between the theoretical and experimental values of $\Phi_{\infty}$ and $\rho_{\infty}$. ${ }^{3,14}$ Note that their theoretical values have been obtained for Gaussian chain without consideration of the fluctuation in hydrodynamic interaction. ${ }^{3}$ Considering this 
fact, it may be concluded that the unperturbed KP theory combined with the QTP theory may give a consistent explanation of the results for the three properties $\left\langle S^{2}\right\rangle,[\eta]$, and $R_{\mathrm{H}}$ for PDiPF.

In Figure 3, the dashed curve represents the unperturbed KP theory values calculated from eq 19 with eq 12 with the abovementioned values of $\lambda^{-1}, M_{\mathrm{L}}$, and $d$. It is seen that the experimental value $(\bigcirc)$ and also the perturbed KP theory one (solid curve) agree with the unperturbed KP theory value in the range of $M_{\mathrm{w}} \lesssim 10^{5}$ but deviate upward progressively from the latter with increasing $M_{\mathrm{w}}$ for $M_{\mathrm{w}} \gtrsim 10^{5}$ due to the intramolecular excluded-volume effect, as in the cases of $[\eta]$ shown in Figure 1 and also of $\left\langle S^{2}\right\rangle{ }^{1}$

\section{Behavior of $\Phi$ and $\rho$}

Finally, we examine behavior of the reduced hydrodynamic volume $\Phi$ and radius $\rho^{-1}$ as functions of $M_{\mathrm{w}}$. In Table IV are given the values of $\Phi$ and $\rho^{-1}$ (and also $\rho$ ) for PDiPF in THF at $30.0^{\circ} \mathrm{C}$ calculated from the present results for $[\eta]$ (Table II) and $R_{\mathrm{H}}$ (Table III) along with the previous results for $\left\langle S^{2}\right\rangle$ (Table I of ref 1).

Figure 4 shows double-logarithmic plots of $\Phi$ (in $\mathrm{mol}^{-1}$ ) and $\rho^{-1}$ against $M_{\mathrm{w}}$ for PDiPF in THF at $30.0^{\circ} \mathrm{C}(\bigcirc)$, a-PS $\left(f_{\mathrm{r}}=0.59\right)$ in cyclohexane at $34.5^{\circ} \mathrm{C}(\Theta)(\triangle),{ }^{14-16,33-35}$ and PHIC in $n$-hexane at $25.0^{\circ} \mathrm{C}(\square) .{ }^{9}$ Both $\Phi$ and $\rho^{-1}$ for a-PS first decrease for $M_{\mathrm{w}} \lesssim 10^{4}$ and then become independent of $M_{\mathrm{w}}$ for $M_{\mathrm{w}} \gtrsim 10^{4}$. This tendency is common to unperturbed flexible polymers. ${ }^{3,36}$ The quantities $\Phi$ and $\rho^{-1}$ are remarkably smaller for PHIC than for a-PS in the range of $M_{\mathrm{w}}$ displayed in this figure, indicating that they decrease with increasing chain stiffness $\left(\lambda^{-1}\right){ }^{3}$ The data points for PDiPF lie between those for a-PS and PHIC, which is consistent with the fact that the chain stiffness of PDiPF $\left(\lambda^{-1}=113 \AA\right)$ is intermediate between those for a-PS $\left(\lambda^{-1}=20.6 \AA\right)$ and PHIC $\left(\lambda^{-1}=\right.$ $840 \AA$ ).

It should be noted here that $\Phi$ and $\rho^{-1}$ become small if the intramolecular excluded-volume effect becomes large. For $\mathrm{PDiPF}$ in $\mathrm{THF}$ at $30.0^{\circ} \mathrm{C}$, however, the effect is not so large in the range of $M_{\mathrm{w}}$ examined (see Figures 1 and 3 ) that $\Phi$ and $\rho^{-1}$ become appreciably smaller than respective unperturbed values. Further, the effect is negligibly small, if any, for PHIC

Table IV. Values of $\Phi, \rho^{-1}$, and $\rho$ for Poly(diisopropyl fumarate) in THF at $30.0^{\circ} \mathrm{C}$

\begin{tabular}{lccc}
\hline sample & $\begin{array}{c}10^{-23} \Phi \\
\left(\mathrm{mol}^{-1}\right)\end{array}$ & $\rho^{-1}$ & $\rho$ \\
\hline PDiPF8 & 1.74 & 0.621 & 1.61 \\
PDiPF9 & 1.53 & 0.671 & 1.49 \\
PDiPF15 & 1.59 & 0.565 & 1.77 \\
PDiPF18 & 1.62 & 0.676 & 1.48 \\
PDiPF22 & 1.49 & 0.641 & 1.56 \\
PDiPF34 & 1.69 & 0.694 & 1.44 \\
PDiPF44 & 1.65 & 0.654 & 1.53 \\
PDiPF50 & 2.01 & 0.680 & 1.47 \\
PDiPF62 & 1.84 & 0.685 & 1.46 \\
PDiPF86 & 1.99 & 0.714 & 1.40 \\
\hline
\end{tabular}

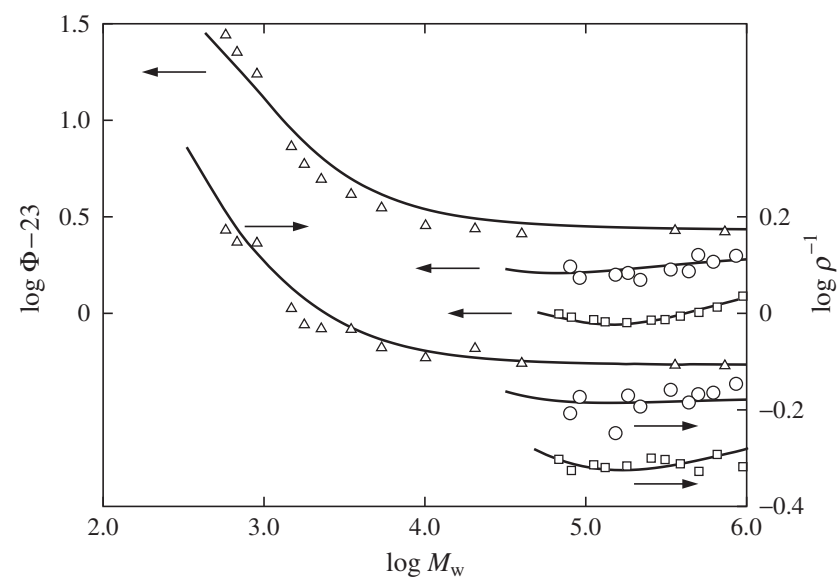

Figure 4. Double-logarithmic plots of $\Phi\left(\right.$ in $\mathrm{mol}^{-1}$ ) and $\rho^{-1}$ against $M_{\mathrm{w}}$ : (○) PDiPF in THF at $30.0^{\circ} \mathrm{C} ;(\triangle)$ a-PS in cyclohexane at $34.5^{\circ} \mathrm{C}$ $(\Theta)$; ${ }^{14-16,33-35}(\square) \mathrm{PHIC}$ in $n$-hexane at $25.0^{\circ} \mathrm{C}$. ${ }^{9}$ The solid curves represent the KP or HW theory values of $\Phi$ and $\rho^{-1}$ (see the text).

in $n$-hexane at $25.0^{\circ} \mathrm{C}$ in the range of $M_{\mathrm{w}}$ displayed in Figure 4. Then the above-mentioned characteristic features in the behavior of $\Phi$ and $\rho^{-1}$ of PDiPF and PHIC may be considered to arise only from their chain stiffness.

In Figure 4, the solid curves associated with the data points for PDiPF represent the perturbed KP theoretical values for $\Phi$ and $\rho^{-1}$ calculated from eq 10 with eq 11 and from eq 21 , respectively, with the perturbed $\mathrm{KP}$ theory values for $[\eta], R_{\mathrm{H}}$, and $\left\langle S^{2}\right\rangle$, where the three sets of the values of the KP model parameters determined from $[\eta]$ and $R_{\mathrm{H}}$ in the present paper and from $\left\langle S^{2}\right\rangle$ and $A_{2}$ in the previous one ${ }^{1}$ have been used in the calculation of [ $\eta], R_{\mathrm{H}}$, and $\left\langle S^{2}\right\rangle$, respectively. The solid curves associated with the data points for a-PS and PHIC represent the unperturbed HW and KP theoretical values, respectively, which have been reproduced from Figure 6.12 of ref 3 . It is seen that the KP and HW theories well explain the difference in the behavior of $\Phi$ and $\rho^{-1}$ between the three polymers.

\section{CONCLUSION}

We have determined $[\eta]$ and $D$ (and $R_{\mathrm{H}}$ ) for PDiPF with $f_{\mathrm{r}}=0.22$ in $\mathrm{THF}$ at $30.0^{\circ} \mathrm{C}$ in the range of $M_{\mathrm{w}}$ from $4.02 \times$ $10^{4}$ to $8.59 \times 10^{5}$. The data so obtained were analyzed on the basis of the corresponding theories for the KP cylinder model combined with the QTP theory for the intramolecular excluded-volume effect. It was shown that the experimental values are in quantitative agreement with the perturbed KP theory ones by the use of the model parameter values consistent with those determined previously ${ }^{1}$ from $\left\langle S^{2}\right\rangle$ and $A_{2}$. The result confirms the previous assertion that the excluded-volume effects must be taken into account in analyses of dilute solution properties of PDiPF in the above-mentioned range of $M_{\mathrm{w}}$ in THF at $30.0^{\circ} \mathrm{C}$. It was also shown that the data points of $\Phi$ and $\rho^{-1}$ for PDiPF lie between those for a-PS (typical flexible polymer) and PHIC (typical semiflexible polymer), reconfirming the validity of the evaluation of the chain stiffness of PDiPF which is intermediate between those of a-PS and PHIC. 
Received: July 10, 2008

Accepted: October 8, 2008

Published: November 19, 2008

\section{REFERENCES}

1. M. Nakatsuji, M. Hyakutake, M. Osa, and T. Yoshizaki, Polym. J., 40, 566 (2008)

2. O. Kratky and G. Porod, Recl. Trav. Chim. Pays-Bas, 68, 1106 (1949).

3. H. Yamakawa, "Helical Wormlike Chains in Polymer Solutions," Springer, Berlin, 1997.

4. H. Benoit and P. Doty, J. Phys. Chem., 57, 958 (1953).

5. H. Yamakawa and W. H. Stockmayer, J. Chem. Phys., 57, 2843 (1972).

6. H. Yamakawa and J. Shimada, J. Chem. Phys., 83, 2607 (1985).

7. J. Shimada and H. Yamakawa, J. Chem. Phys., 85, 591 (1986).

8. H. Yamakawa, Macromolecules, 25, 1912 (1992).

9. H. Murakami, T. Norisuye, and H. Fujita, Macromolecules, 13, 345 (1980).

10. F. Abe, Y. Einaga, T. Yoshizaki, and H. Yamakawa, Macromolecules, 26, 1884 (1993).

11. T. Otsu, H. Minai, N. Toyoda, and T. Yasuhara, Makromol. Chem., Suppl., 12, 133 (1985).

12. T. Otsu, T. Yasuhara, and A. Matsumoto, J. Macromol. Sci. Chem., A25, 537 (1988).

13. A. Matsumoto and E. Nakagawa, Euro. Polym. J., 35, 2107 (1999).

14. T. Konishi, T. Yoshizaki, and H. Yamakawa, Macromolecules, 24, 5614 (1991).

15. T. Yamada, T. Yoshizaki, and H. Yamakawa, Macromolecules, 25, 377 (1992).

16. F. Abe, Y. Einaga, and H. Yamakawa, Macromolecules, 26, 1891 (1993).
17. F. Abe, K. Horita, Y. Einaga, and H. Yamakawa, Macromolecules, 27, 725 (1994).

18. M. Kamijo, F. Abe, Y. Einaga, and H. Yamakawa, Macromolecules, 28, 1095 (1995).

19. K. Horita, N. Sawatari, T. Yoshizaki, Y. Einaga, and H. Yamakawa, Macromolecules, 28, 4455 (1995).

20. Y. Tominaga, I. Suda, M. Osa, T. Yoshizaki, and H. Yamakawa, Macromolecules, 35, 1381 (2002).

21. T. Arai, F. Abe, T. Yoshizaki, Y. Einaga, and H. Yamakawa, Macromolecules, 28, 3609 (1995).

22. M. Osa, F. Abe, T. Yoshizaki, Y. Einaga, and H. Yamakawa, Macromolecules, 29, 2302 (1996).

23. T. Arai, N. Sawatari, T. Yoshizaki, Y. Einaga, and H. Yamakawa, Macromolecules, 29, 2309 (1996).

24. H. Yamakawa and M. Fujii, Macromolecules, 7, 128 (1974).

25. H. Yamakawa and T. Yoshizaki, Macromolecules, 13, 633 (1980).

26. H. Yamakawa, "Modern Theory of Polymer Solutions," Harper \& Row, New York, 1971. Its electronic edition is available on-line at the URL: http://www.molsci.polym.kyoto-u.ac.jp/archives/redbook.pdf

27. A. J. Barrett, Macromolecules, 17, 1566 (1984).

28. H. Yamakawa and M. Fujii, Macromolecules, 6, 407 (1973).

29. H. Yamakawa and T. Yoshizaki, Macromolecules, 12, 32 (1979).

30. H. Yamakawa and T. Yoshizaki, Macromolecules, 28, 3604 (1995).

31. A. J. Barrett, Macromolecules, 17, 1561 (1984).

32. C. Domb and A. J. Barrett, Polymer, 17, 179 (1976).

33. Y. Einaga, H. Koyama, T. Konishi, and H. Yamakawa, Macromolecules, 22, 3419 (1989).

34. T. Arai, F. Abe, T. Yoshizaki, Y. Einaga, and H. Yamakawa, Macromolecules, 28, 5458 (1995).

35. T. Konishi, T. Yoshizaki, T. Saito, Y. Einaga, and H. Yamakawa, Macromolecules, 23, 290 (1990).

36. I. Suda, Y. Tominaga, M. Osa, T. Yoshizaki, and H. Yamakawa, Macromolecules, 33, 9322 (2000). 